\title{
Triangulation positioning system network
}

\author{
Marios Sfendourakis ${ }^{1}$, Rajagopal Nilavalan ${ }^{1}$, and Emmanuel Antonidakis ${ }^{2}$ \\ ${ }^{1}$ University of Brunel - Dept of Electronic and Computer Engineering, UB8 3PX Kingston \\ Lane, Uxbridge Middlesex United Kingdom \\ ${ }^{2}$ Technological Educational Institute of Crete (TEI of Crete) - Dept of Electronic Engineering, \\ Romanou 3 Chalepa Greece
}

\begin{abstract}
This paper presents ongoing work on localization and positioning through triangulation procedure for a Fixed Sensors Network - FSN.The FSN has to work as a system.As the triangulation problem becomes high complicated in a case with large numbers of sensors and transmitters, an adequate grid topology is needed in order to tackle the detection complexity.For that reason a Network grid topology is presented and areas that are problematic and need further analysis are analyzed. The Network System in order to deal with problems of saturation and False Triangulations - FTRNs will have to find adequate methods in every sub-area of the Area Of Interest - AOI.Also, concepts like Sensor blindness and overall Network blindness, are presented. All these concepts affect the Network detection rate and its performance and ought to be considered in a way that the network overall performance won't be degraded.Network performance should be monitored contentiously, with right algorithms and methods. It is also shown that as the number of TRNs and FTRNs is increased Detection Complexity - DC is increased.It is hoped that with further research all the characteristics of a triangulation system network for positioning will be gained and the system will be able to perform autonomously with a high detection rate.
\end{abstract}

Keywords - Fixed Stations Network, Triangulation, Localization, Network Blindness, Triangulation Area.

\section{INTRODUCTION}

The problem of localization is under research last years, whereas the applications of Sensors Networks are spread year by year in many fields, ways to find the position of a Sensor or a Transmitter with high accuracy are still tested and remain of high importance. One of the localization techniques is the process of Triangulation which was analyzed in [1]. It was also shown that in a large network with a great number of Sensors (SRs) there are cases of pseudo transmitters (PTRNs) which need to be examined on a case by case basis. The goal of this paper is to shed light on various issues that should be put into consideration when a Sensors Network (SN) has to work as an automated system. Various cases will be analyzed and it will be shown that for an automated network with many SRs and a great number of TRs that operate in an area of interest, there are attributes that need great attention and need to be examined and integrated in the system. A fixed stations network isn't able to move it's SRs but with logical programming it can analyze and combine the SRs data and provide valuable results. When results with high accuracy are collected the positions of new transmitters that entered the A OI will be acquired.So far,bearing only estimation is one of the fundamental and challenging problems in target tracking and Multisensor-multitarget bearing-only tracking is a challenging problem with many applications [5],[6],[7].

\section{Contributions}

Prior research has yielded mixed findings mainly related with SR to SR position in Wireless Sensor Networks WSNs and WSNs with mobile SRs. The main problem of that research is not to find the SRs positions after their random deployment in an area.In our research we focus in finding new TRs positions who enter a Fixed Sensors Network - FSN area, with known positions of SRs, and with an initial state of TRs positions in that area. We show that a FSN, in order to localize TRs in an initial state and being able to tracking the new TRs in a new state the network should focus in processing of SR to SR data and each SR's state. Not only a SR might become blinded by degrading the detection performance in an area but also the overall state of the network might become blinded or saturated.It is also showed that the network ought to combine various ways in order to remain in a high detection performance.In addition, network detection rate and network detection performance are presented for this type of network and its function for transmitters - TRs detection. 


\section{Sensors Blindness}

Every Sensor- SR in the network will collect some data of Bearings-BRNGs which are related with the Transmitters-TRs that will be detected in one area. Each SR has a circular region with radious $r$ for detection. The problem is that as the number of existing TRs increase then the SRs become blind and many new TRs in it's area of responsibility might not be detected. As it is depicted in Fig. 1 the SR1 and SR2 have a number of bearings which are black colored. The fact is that if a new TR enter a SR's radius, in a bearing similar with the previous, then it might only be detected by another SR. In Fig.2a SR1 and SR2 have certain sets of BRNGs. Then two new TRs appear in the area, TR3 and TR4 which are detected by SR2 Fig.2b.SR1 has already BRNGs at those radials.

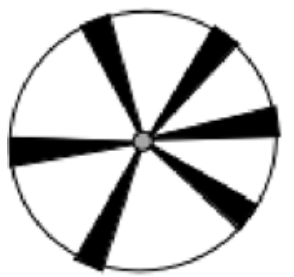

SR1

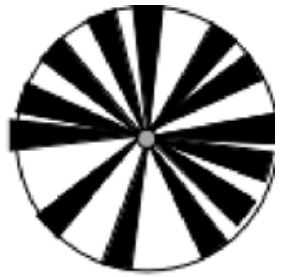

SR2
Figure 1

For that reason we have to define a SR parameter that affects the detection through TRN and which is the Sensor Blindness - $\mathrm{SR}_{\mathrm{BL}} \cdot \mathrm{SR}_{\mathrm{BL}}$ is related with the number of BRNGs already acquired and the SR detection accuracy error.We take for granted an average network error for detection for each $\mathrm{SR}, \mathrm{S}_{\mathrm{ER}}$ which might be $2^{0}$ to $5^{0}$ degrees.

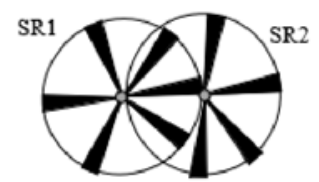

Figure 2a

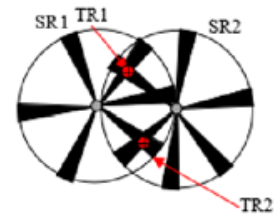

Figure 2b
So,Sensor Blindness $\mathrm{SR}_{\mathrm{BL}}$ is defined as:

$$
\mathrm{SR}_{\mathrm{BL}}=\mathrm{R}_{\mathrm{SRs}} \times 2 \mathrm{~S}_{\mathrm{ER}} / 360 * 100 \%
$$

For example ,if a SR has 6 BRNGs $(30,60,120,224,330,10)$ and the network has an average detection error for each SR, $4^{0}$ then the SR blindness will be:

$$
\text { SR1 Blindness : } 6 \text { x (2x 4) / } 360 * 100=13.33 \%
$$

That means that at a percentage of $13.33 \%$, SR1 will not detect a new TR but only another SR,within its region will find that new TR.In Fig.1, SR2 has a high level of blindness of $28.8 \%$.

\section{Network Parameters}

An FSN in order to be operational a lot of parameters should be examined and checked not only before it's operation but also when it operates. SRs characteristics should be checked and also it's topology need to be carefully designed as problematic areas might degrade it's performance.Areas of TRN whilst will detect TRs ,synchronously will saturate it's state. For that reason parameters like Network Blindness, Network Saturation, Network Detection rate etc. should be approached.

\subsection{Network Blindness}

As it was previously mentioned, every SR of the network has a level of blindness. Summing the blindness of all the SRs then we have a picture of the network Blindness, which is a factor that characterizes the detection performance of the network.In Fig. 3 we see a grid network with SRs that have a low level of blindness.We see that there exist enough free area for detection. Comparing Fig. 3 with Fig. 4 we see that in Fig. 4 the free area for detection is far more less, and by that way the process of detection is more complicated. Network Blindness Definition $-\mathrm{N}_{\mathrm{BL}}$ where $\mathrm{n}$ is the number of $\mathrm{SR}_{\mathrm{s}}$

$$
\mathrm{N}_{\mathrm{BL}}=\mathrm{B}_{1}+\mathrm{B}_{2}+\ldots+\mathrm{Bn} / \mathrm{n}
$$

(Example $1 \quad$ Sensor Network Blindness)

We have a Network of 4 SRs with various BRNGs,[SR1 $4(170,200,320,46)$, SR2 $6(30,60,120,224,330,10)$, ,SR3 3 (210,

240,300), SR4 2(18,70). $\quad$ SRs Average Error $=4$.

The total of SRs BRNGs is:

BRNGs Total : $4+6+3+2=15$ BRNGs

$\mathrm{N}_{\mathrm{BL}}$ is the sum of all the SRs blindness of the network divided with the number of SRs- $\mathrm{N}_{\mathrm{SRs}}$.

$$
\begin{array}{r}
\mathrm{N}_{\mathrm{BL}}=\mathrm{B}_{1}+\mathrm{B}_{2}+\mathrm{B}_{3}+\mathrm{B}_{4} / \mathrm{N}_{\mathrm{SRs}} \\
\mathrm{N}_{\mathrm{BL}}=13,33+16,4+10+6 / 4=11,43
\end{array}
$$

So,in this case the overall Network Blindness is:

$\mathrm{N}_{\mathrm{BL}}=11,43 \%$ 

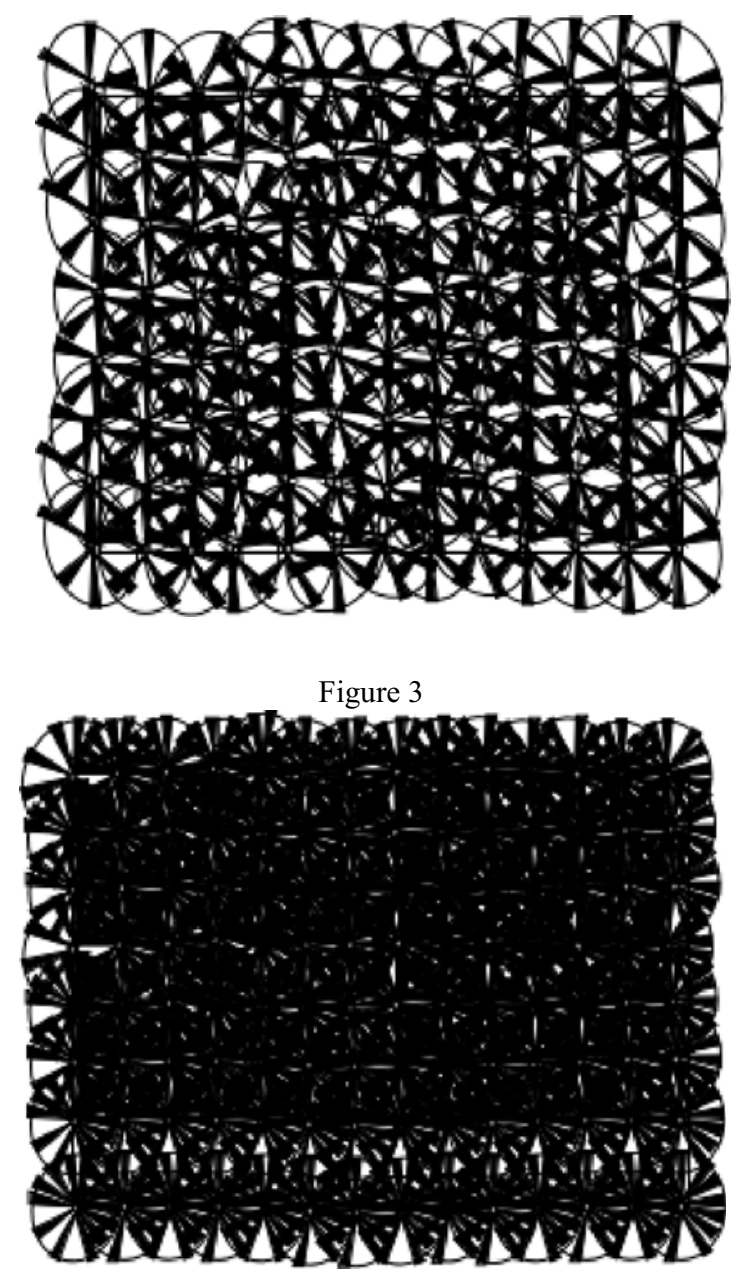

Figure 4

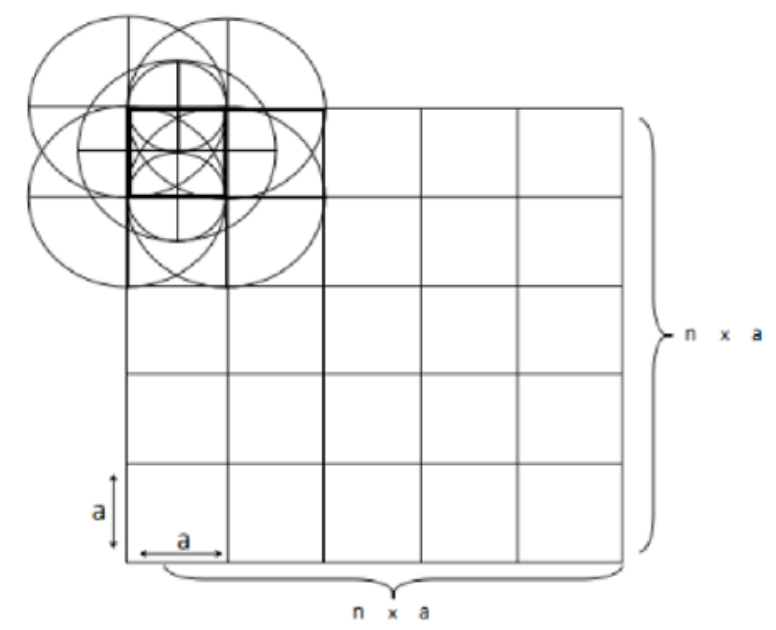

Figure 5

Although the distance between SR4 and TR1 is high, the TR1 is detected due to its high power. A TR which isn't detected due to its low power, will be detected by other SRs which are at close distance.

\subsection{Network Saturation}

Another attribute of the network which need further analysis is the number of $\mathrm{TRN}_{\mathrm{s}}$. As the number of TRNs in the network AOI increases, then the network approaches a saturation state that degrades it's performance.We define the network saturation state with the following parameters:

Network Area Saturation State: $\mathrm{N}_{\mathrm{AST}}$

Area Size: $A_{S}$

$$
\mathrm{N}_{\mathrm{AST}}=\text { Total Area of TRNs /Area Size }
$$

So, $\mathrm{N}_{\text {TAST }}$ is given by the equation (1),

$$
\mathrm{N}_{\mathrm{AST}}=\sum_{i=1}^{n} \mathrm{TRNi} / \mathrm{A}_{\mathrm{S}}
$$

Where $\mathrm{n}$ is the number of TRNs of the network in a current state.In order to deal with the various cases of TRNs the system has to find the number of TRNs in each sub-area in the grid and then process the Network Area Saturation State - $\mathrm{N}_{\mathrm{AST}}$, for that sub-area. Assuming that an AOI of the Network has $\mathrm{n}$ sub-areas, then the Total Network Saturation State, $\mathrm{N}_{\mathrm{TAST}}$ is given by the equation (2),

$$
\mathrm{N}_{\mathrm{TAST}}=\sum_{i=1}^{n} \mathrm{~N}_{\mathrm{ASTi}} / \mathrm{A}_{\mathrm{TS}}
$$

where $A_{T S}$ is the total area size.

\subsection{Network Detection rate}

Another parameter that need to be defined for a FSN for localization via triangulation is the Network Detection Rate - $\mathrm{ND}_{\text {rate }}$. We define $\mathrm{ND}_{\text {rate }}$ with the equation (3):

$\mathrm{ND}_{\text {rate }}=100-\left[\left(\operatorname{SRD}_{\mathrm{F}}+\mathrm{NB}_{\mathrm{BL}}(2-\mathrm{k})\right)+\mathrm{ETRN}_{\mathrm{s}}\right] / 3$

where ETRN $_{\mathrm{s}}$ is the number of Existing Triangulations. We define $\mathrm{k}$ as a quotient', $\mathrm{k}=1 / \mathrm{ETRN}_{\mathrm{s}}$, where: $0<\mathrm{k}<1$, assuming that as the number of $\mathrm{ETRN}_{\mathrm{s}}$ increase, then $\mathrm{N}_{\mathrm{BL}}$ is affected. $\mathrm{SRD}_{\mathrm{F}}$ is the Sensors Detection Failure and it is the number of SRs of the Network which will fail to detect TRs.

\subsection{Network Grid topology}

In Fig. 5 ,it is depicted the Area of Interest - AOI for detection.In this area we see a grid perspective of squares where clusters of SRs cover the whole area and form regions of coverage. In each individual square there are seven circles which cover each sub-region, Fig.6. 


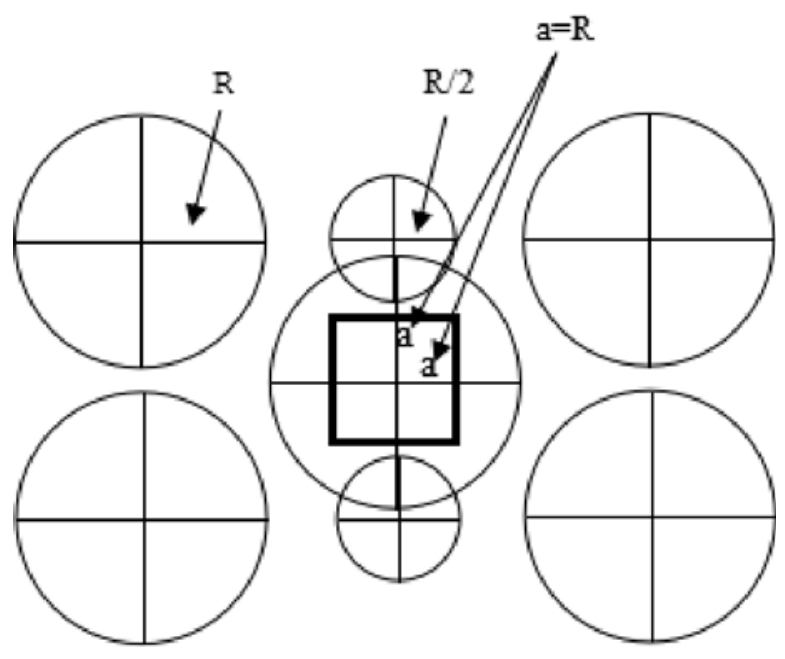

Figure 6

Their coverage intersect with each other and by that way the whole area is monitored, Fig.8.Each circle is the peripheral area of coverage of a SR. We have two types of SRs. One type that has a circular coverage of radius $r$, where $r=a$, and a second type with a circular coverage of radius $r / 2=a / 2$. By that way, SRs with a small radius coverage are minimizing the area of TRN in each square. Additionally, SRs with radius coverage can monitor four square regions by each of their four quadrant, except circles that are located in the peripheral area of the grid which monitor two squares.Circles located at the AOI grid corners monitor only one quadrant.In the following Fig. 7 we have a depiction of a Grid with twenty five subareas.

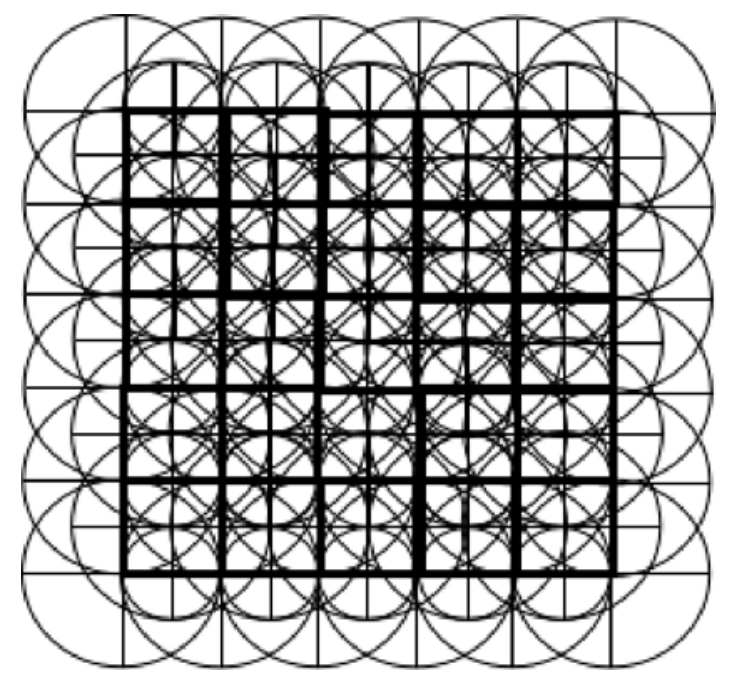

Figure 7

Each sub-area is monitored by the seven SRs and an additional SR that overlaps the area. A similar splitting procedure is applied in a GSM network, where an area is monitored by TRs that operate in hexagonal cells as it is depicted in Fig.9.The actual radio coverage of a cell is called footprint. The radio network operates in a bundle of frequencies, in this case (A,B,C,D,E,F,G) [2],[3],[4].

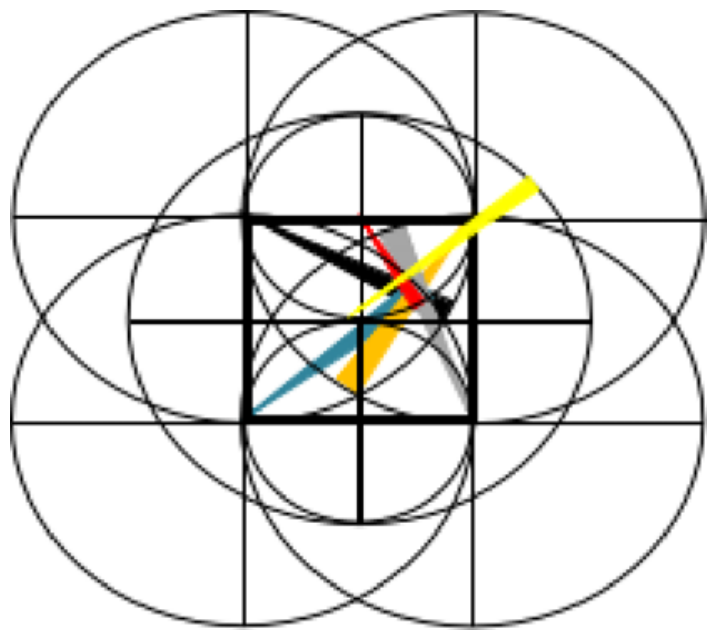

Figure 8

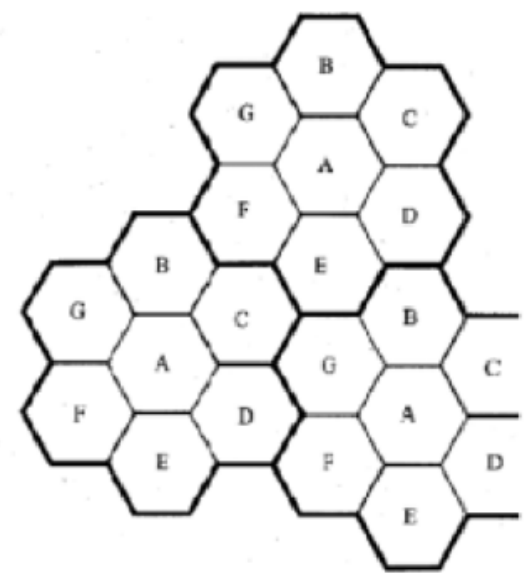

Figure 9

The size of cells might vary and often the size of cells might be splitted. By Cell splitting Fig.10, it is achieved capacity improvement by rescaling the system and the number of times that channels are reused is increased. Due to smaller cells, capacity increases and that is due to additional number of channels per unit area [3].
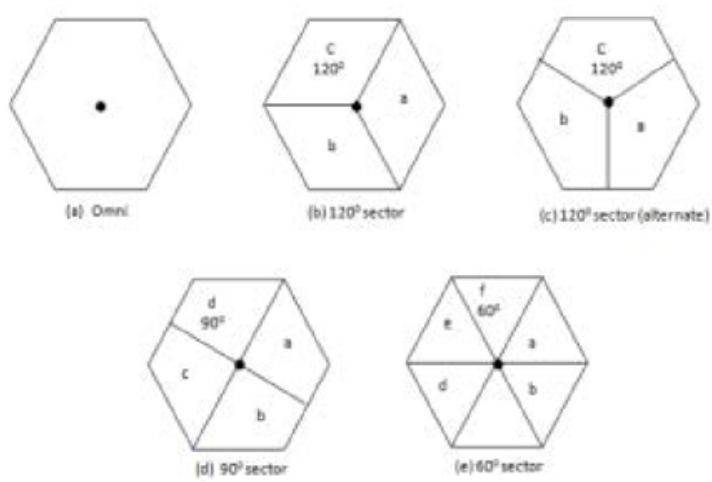

Figure 10

In the Fixed Station Network - FSN the area is monitored by SRs that operate in the same frequency 
whilst vary in sensitivity. During processing of data acquired, every area is processed independently from the other.All the parameters of the network are also acquired and at the same time the SRs parameters are checked. If inside an area a SR has problem of high blindness then its operation might be degraded. Thresholds of blindness have to be applied for each SR.

\subsection{Network Detection Performance}

In the following figures, Fig.11 initial State, and Fig.12, actual State 1,TRNs are depicted with red color FTRNs with black and new TRNs with green color.As the number of TRNs and FTRNs is increased Detection Complexity - DC is increased. We define DC as the level of easiness to detect a new TR in the area.The system has to deal with the detection of new TRs while the areas of TRNs and false TRNs that probably exist, have to be tackled on a case by case basis. There is a high probability that the number of TRNs and FTRNs will be increased, (in Fig.12 we see that TRNs and FTRNs are increased) and that presupposes that every area should be monitored by a minimum number of SRs. These SRs should be able to find a new TR and synchronously reject a FTRN.In Fig.12 it is depicted the actual state 1 which is a problematic area with FTRNs and new TRs that entered the area. The system will detect the new TRs with a detection algorithm which we name Sensors Cluster Detection - SCD. Its name implies that Clusters of SRs are working as groups to process one area and seek for new TRs.This algorithm will be presented and analyzed in a future paper.A problematic situation appears when SRs have a high rate of saturation which also means that the area is saturated. For these reasons, like in the case of Centralized Intrusion Detection Systems - CIDS where a centralized computer monitors all the activities in the network and detects intrusions by analyzing the monitored network activity data. [8] SRs Clusters should monitor each area and send their data to a central computer. Data fusion and adequate data analysis should provide results with high accuracy.The problem of target tracking with the process of triangulation deteriorates more in the case that exist many TRNs which block detection beyond the area of TRN.

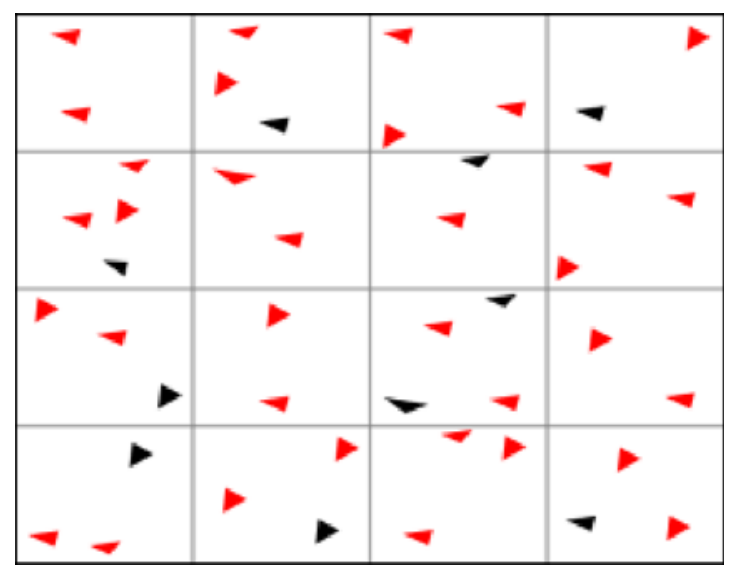

Figure 11

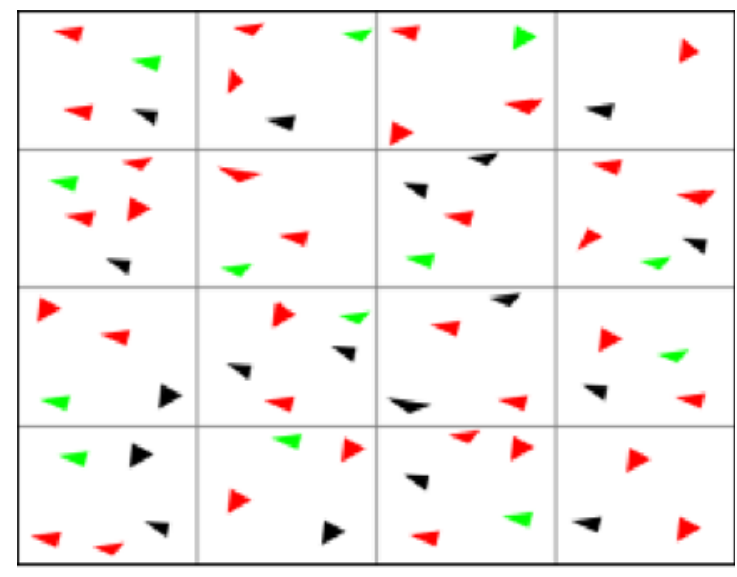

Figure 12

\section{References}

[1] M.Sfendourakis, N.Rajagopal, E.Antonidakis "Automatic Triangulation Positioning System for wide area coverage from a Network of Stations in Fixed Positions". In Proceedings of the $19^{\text {th }}$ International Conference on Systems (part of CSCC '15), Recent advances in Systems (2015): (pp.650-660).

[2] R.P. Yadav et.al, 'Wireless Communication', 2008.

[3] Nura Muhammad Shehu 'Coverage and Capacity Improvement in GSM Network' [IJNREME -2015 ] 2, pp: (57-62).

[4] Patel, Sheshhan R., and A. K. Sisodia. "Techniques for Improving Channel Capacity of Downlink in GSM Systems." (2014).

[5] E. Taghavi, R. Tharmarasa, T. Kirubarajan, and M. McDonald. Multisensor multitarget bearing only sensor registration". IEEE Transaction on Aerospace and Electronic Systems, vol. 52, no. 4, August 2016.

[6] Y. Bar-Shalom, X. R. Li, and T. Kirubarajan, Estimation with Applications to Tracking and Navigation: Theory, Algorithms and Software, Wiley, NY,2001.

[7] Y. Bar-Shalom, P. K. Willett, and X. Tian, Tracking and Data Fusion: A Handbook of Algorithms, YBS Publishing, Storrs, CT, 2011.

[8] Butun, I., Morgera, S.D. and Sankar, R., 2014. A survey of intrusion detection systems in wireless sensor networks. IEEE Communications Surveys \& Tutorials, 16(1), pp.266-282. 